\title{
Der Mythos der schnellen Integration Eine empirische Untersuchung zur Integration der Vertriebenen und Flüchtlinge in der Bundesrepublik Deutschland bis 1971*
}

\author{
Paul Lüttinger \\ Institut für Sozialwissenschaften, Universität Mannheim, Tattersallstr. 2, D-6800 Mannheim 1
}

Zusammenfassung: Auf der Materialbasis der Mikrozensus-Zusatzerhebung „Berufliche und soziale Umschichtung der Bevölkerung" vom April 1971 werden die Konsequenzen von Flucht und Vertreibung nach dem Zweiten Weltkrieg für die Übernahme der Vertriebenen und Flüchtlinge in das Beschäftigungssystem der Bundesrepublik untersucht. Ausgangspunkt der Untersuchung ist die Hypothese, daß sich die ungleichen Chancen der Vertriebenen und Flüchtlinge schnell abschwächten und in der Gegenwart (d. h. zum Befragungszeitpunkt 1971) keine Differenzen zur einheimischen Bevölkerung mehr vorhanden sind. Die vergleichende Analyse der einheimischen mit der zugezogenen Bevölkerung zeigt jedoch, daß die Generalhypothese einer chancenindifferenten Integration der Zuwanderer nicht bestätigt werden kann. Es läßt sich stattdessen eine erhebliche selektive Wirkung des Strukturwandels auf die beruflichen Chancen der Ansässigen gegenüber der neuen Bevölkerung nachweisen.

\section{Problemstellung und Einleitung}

Schon während des Zweiten Weltkrieges begann eine der größten gewaltsamen Umsiedlungsaktionen in der Geschichte der Neuzeit. Die 1944 einsetzende Ost-West-Wanderung beeinflußte mannigfaltig die Entwicklung und Zusammensetzung der Sozialstruktur der Bundesrepublik. Allein die Bevölkerung nahm von 1939 (Gebietsstand BRD) bis 1971 um ca. 19 Millionen Einwohner zu. Der Hauptanteil dieses Wachstums wurde bis 1961 vor allem über Wanderungsgewinne durch Vertriebene und Flüchtlinge erzielt, deren Anzahl 1970 ca. 14,7 Millionen betrug, also nahezu ein Viertel der Wohnbevölkerung der Bundesrepublik. Zum Vergleich: Der Anteil der Ausländer an der Wohnbevölkerung der Bundesrepublik betrug 1984 ca. 7,6\% (4,6 Millionen).

Die Probleme der Nachkriegszeit, die durch Kriegszerstörungen und Zuwanderungswellen kumulierten, fanden ihren Niederschlag sowohl in institutionellen Einrichtungen (wie dem Ministerium für Vertriebene, Flüchtlinge und Kriegsgeschädigte), zahlreichen Verbänden als auch in einer großen Zahl von gesetzlichen Regelungen seit der Konstituierung der Bundesrepbulik 1949 bis zur Regierung Brandt/Scheel. Dennoch existierten auch damals Spannungen und Konflikte zwischen Zuwanderern und Einheimischen, die teilweise

* Für die kritische Durchsicht des Manuskripts und hilfreiche Anregungen danke ich Prof. Dr. Walter Müller. Rita Rossman fertigte mit großer Sorgfalt die Schaubilder an. von einem geradezu „klassenkämpferischen Geist“ beherrscht wurden (Grosser 1982: 273).

Mit dem Wiederaufbau ,geht der Abbau der Spannungen zwischen den Bevölkerungsteilen einher" (Mackensen 1979: 499). Spätestens jedoch mit der Auflösung des Bundesministeriums für Vertriebene und Flüchtlinge 1969 - einzelne Ressorts gingen auf das Ministerium für gesamtdeutsche Fragen über - wurde dokumentiert, daß man die wirtschaftliche und soziale Integration der Zuwanderer als vollzogen betrachtete. Der vorherrschenden öffentlichen und auch wissenschaftlichen Meinung nach war das Integrationsproblem ohnehin seit Mitte der fünfziger Jahre mit der erreichten Vollbeschäftigung obsolet geworden und auch die $\mathrm{kul}$ turelle Integration galt als weitgehend abgeschlossen (Vogelsang 1975: 174; Henning 1975: 210). ${ }^{1}$

Die zunehmende kulturelle und auch politische Integration (d. h. der Bedeutungsverlust der Vertriebenenparteien bei Wahlen) bei gleichzeitigem Wirtschaftswachstum, Wohlstandssteigerung und

\footnotetext{
${ }^{1}$ Somit erstaunt es nicht, daß die Anzahl (auch neuerer) sozialwissenschaftlicher Untersuchungen zum Integrationsproze $\beta$ relativ gering ist, so daß man durchaus von einer gewissen Abneigung und Nichtbeachtung dieses Komplexes auch von seiten der Soziologie sprechen kann (Waldemann 1979: 163). Vor allem wurde bislang kaum systematisch untersucht, welchen Anteil die $\mathrm{Zu}$ wanderer am sozialen Wandel nach dem Zweiten Weltkrieg hatten (vgl. hierzu Steiger \& Tegtmeyer 1975), d. h. - auf deskriptiver Ebene - inwieweit sich der vollziehende Strukturwandel bei Vertriebenen, Flüchtlingen und Einheimischen gleichermaßen abbildete.
} 
das Ausbleiben der befürchteten Radikalisierung der Zuwanderer erlaubt jedoch noch nicht den Schluß auf eine erfolgreiche soziale Integration. ${ }^{2}$ Ein großer Anteil am Abbau der Spannungen und Probleme wird - neben der Bereitstellung von Arbeitsplätzen - dem Lastenausgleich (bzw. dem Soforthilfegesetz von 1949) zugeschrieben. Der Lastenausgleich, der erst nach langen Diskussionen und parteiinternen Auseinandersetzungen verabschiedet wurde, vermittelte neben den finanziellen Transferleistungen natürlich auch auf subjektiver Ebene das Gefühl der sozialfürsorgerischen Betreuung durch die Behörden. In der Tat brachte er zwar einen hohen Vermögenstransfer (bis 1979 über 114 Mrd DM), führte jedoch keineswegs zu einer Umverteilung oder Änderung der Vermögensverhältnisse. Dennoch kann der Lastenausgleich pars pro toto als eine der wichtigsten gesetzlichen Bestimmungen und als Versuch betrachtet werden, die Problematik der Existenzgründung und der sozialen Integration zu bewältigen - wenngleich den Sozialleistungen in finanzieller Hinsicht mehr Bedeutung zukamen. Daneben wurde die Absicht verfolgt, die befürchtete politische Destabilisierung zu verhindern (vgl. Schillinger 1985). Obwohl dem Argument, daß sich die wirtschaftliche und soziale Integration ohne Lastenausgleich weitaus problematischer vollzogen hätte, nicht widersprochen werden soll, muß der Effekt dieser Bemühungen auf einer gesamtgesellschaftlichen Ebene durchaus kritisch gesehen werden. Wie später noch deutlich wird, zeigen gerade die Zuwanderer, die mit zu den Hauptzielgruppen der gesetzlichen Bemühungen zählten, in einer langfristigen Perspektive gegenüber den Einheimischen deutlich anders verlaufende strukturelle Entwicklungsmuster.

${ }^{2}$ Zur deskriptiven Erfassung der Auswirkungen von Flucht und Vertreibung wurde ein ,sozialstruktureller ${ }^{`}$ Integrationsbegriff verwendet. Zum einen da die der Untersuchung zugrunde liegende Materialbasis (Mikrozensus-Zusatzerhebung „Berufliche und soziale Umschichtung der Bevölkerung" vom April 1971) keine subjektiven Angaben zur Erfassung des Integrationsvorganges enthält; zum anderen da die gegenwärtigen zentralen Integrationsmodelle auf handlungstheoretischen Prämissen beruhen und per definitionem Zwangswanderungen aus den Modellüberlegungen ausschließen. Die Zuwanderer werden dann als integriert betrachtet, wenn sie a) eine den Einheimischen entsprechende Entwicklung in ihrer sozialen strukturellen Ausdifferenzierung aufweisen und b) sich für die Zuwanderer die gleichen Chancenmuster wie für die Einheimischen ergeben.
Im folgenden wird versucht, die gleichzeitig stattfindenden Entwicklungen - Integrationsverlauf und struktureller Wandel - anhand von sozioökonomischen Indikatoren darzustellen. Dabei sollte jedoch beachtet werden, daß diese Indikatoren nicht nur technisch-wirtschaftliche Entwicklungen dokumentieren. Gleichermaßen wichtig waren sicherlich ebenso Bemühungen zur politischen und gesellschaftlichen Legitimation von Ansprüchen aufgrund des kollektiven Schicksals und die damit verbundenen sich ändernden Einstellungen und Werthaltungen aller betroffenen Bevölkerungsteile. ${ }^{3}$ Ebenso bleiben elementare Bereiche wie Wohnverhältnisse, Arbeitsbedingungen, soziale Kontakte etc. aus den folgenden Betrachtungen ausgeklammert, obwohl auch diese in hohem MaBe Integrationsprozesse determinieren (Hoffmann-Nowotny/Hondrich 1981).

\section{Begriffliche Definitionen und Datenbasis ${ }^{4}$}

Der Gesetzgeber unterscheidet im Zusammenhang mit Flucht und Vertreibung zwei Hauptgruppen (vgl. Bundesvertriebenengesetz vom 19. 5. 1953 $\S 1-20$ ): Flüchtlinge, wobei der Begriff ausschließlich auf die Zuwanderer aus der SBZ/DDR angewendet wird (Flüchtlingsausweis $C$ ), und Vertriebene für alle aus den ehemaligen Ostgebieten $\mathrm{Zu}$ gewanderte. Bei der Kategorie ,Vertriebene' erfolgt eine weitere Differenzierung in ,Heimatvertriebene' die ihren Wohnsitz am 1. 1. 1937 in den Ostgebieten hatten (Vertriebenenausweis A) und ,Vertriebene", die am 1. 1. 1939 ihren Wohnsitz in den ehemals deutschen Ostgebieten hatten (Vertriebenenausweis $\mathbf{B}$ ).

Das Bundesvertriebenengesetz kennt jedoch nicht nur den originären Erwerb des Vertriebenen- oder Flüchtlingsstatus, sondern auch den abgeleiteten oder zugeschriebenen durch Geburt. Nach der Vertreibung geborene Kinder erwerben die Eigenschaft des Elternteils, dem zum Zeitpunkt der

${ }^{3}$ So kann auch an dieser Stelle die sicherlich wichtige Position und Funktion der Landsmannschaften und Vertriebenenverbände nicht diskutiert werden (vgl. hierzu Schoenberg 1970). Die unterschiedliche geographische Konzentration der Zuwanderer und die damit verbundene unterschiedliche Höhe der sozialen Probleme - ein weiteres sozial relevantes Trennungskriterium - muß ebenfalls unberücksichtigt bleiben.

${ }^{4}$ Die Verwendung der Begriffe erfolgte bis 1953 keineswegs einheitlich und war vielen Änderungen unterworfen. 
Geburt oder Legitimation das Recht auf Personensorge zusteht. Dies führt mit zunehmendem Abstand vom Zeitpunkt der Wanderungen zur $\mathrm{Zu}$ nahme der Zahl von Vertriebenen und Flüchtlingen, die diese Ereignisse nicht mehr als persönliches Schicksal erlebten. 1974 waren 31,4\% der Vertriebenen und 29,5\% der Flüchtlinge nach 1949 geboren (Statistisches Bundesamt 1975).

Für die vorliegende Untersuchung wurden unter die Bezeichnung Vertriebene sowohl Vertriebene mit Ausweis A und B als auch Wohnsitzvertriebene subsumiert. Die Kategorie Flüchtlinge kennzeichnet Zugezogene aus der SBZ/DDR sowohl mit als auch ohne Flüchtlingsausweis, d. h. Zuwanderer, die einen Antrag auf Anerkennung stellten und auch anerkannt wurden (Flucht aufgrund besonderer Zwangslagen im Sinne von Gefahr für Leib und Leben oder persönliche Freiheit) als auch sonstige Zuwanderer aus der SBZ/DDR, die keinen Antrag stellten oder nicht anerkannt wurden. Die Bezeichnung Zuwanderer wird im folgenden für beide Kategorien (Flüchtlinge und Vertriebene) benutzt. Als Einheimische sind Personen definiert, die schon vor dem Zweiten Weltkrieg, am 1. 1. 1939 ihren ständigen Wohnsitz auf dem Gebiet der Bundesrepublik hatten.

\section{Datenbasis}

Grundlage der Untersuchung ist die vom Statistischen Bundesamt durchgeführte Mikrozensus-Zusatzerhebung vom April 1971 „Berufliche und soziale Umschichtung der Bevölkerung“" (MZU 71), eine repräsentative $1 \%$-Stichprobe von Personen der deutschen Wohnbevölkerung, die 1956 und früher geboren wurden und somit zum Erhebungszeitpunkt 15 Jahre und älter waren. ${ }^{5}$

Mit der MZU 71, die eigens zur Erfassung des Integrationsprozesses bereits Mitte der sechziger Jahre geplant wurde, sind in dieser Form erstmals reichhaltige Daten zur Sozialstruktur erhoben worden, wobei aufgrund des speziellen Erkenntnisinteresses nicht nur die einheimische Bevölkerung, sondern auch die durch die Ereignisse des Zweiten Weltkrieges Vertriebenen und Flüchtlinge befragt wurden. ${ }^{6}$

5 Zur Entstehungsgeschichte und Durchführung vgl. Tegtmeyer 1976: 4-34.

${ }^{6}$ Hinsichtlich des ursprünglichen Anlasses erfolgten bislang kaum Analysen mit der MZU 71, in denen die Zuwanderer explizit als Untersuchungseinheit berücksichtigt wurden (vgl. jedoch Tegtmeyer/Steiger 1975; Rauch 1979).
Die Stichprobengröße beträgt insgesamt 209262 (männliche) Personen. Darunter befinden sich 38211 Ausweis- und Wohnsitzvertriebene $(18,2 \%), 10555$ Flüchtlinge $(5 \%)$ und 160496 Einheimische $(76,8 \%)$. Der Zuwandereranteil an der Gesamtstichprobe beträgt somit $23,2 \%(\mathrm{~N}=$ 48766 ).

\section{Die sozioökonomische Entwicklung der Bundesrepublik bis 1971}

Durch die millionenfache Zuwanderung von Vertriebenen, Flüchtlingen und Kriegsheimkehrern sowie durch die Abtrennungen der Ostgebiete des Deutschen Reichs veränderte sich das Bild Nachkriegsdeutschlands innerhalb kurzer Zeit erheblich.

Einige wenige Beispiele aus einer Vielzahl von Kriegsfolgen sind das Ansteigen der Bevölkerungsdichte auf dem Gebiet der Bundesrepublik von 160 Einwohnern je qkm (1939) auf 218 Einwohner je qkm bis 1961 (Bethlehem 1982: 28), die Verringerung der quantitativen Minderheitenlage der Katholiken, die Abspaltung erheblicher Wählerpotentiale der SPD (Lepsius 1974: 264; Dahrendorf 1977: 131f), das überproportionale Bevölkerungswachstum der Landkreise, das durch ungleiche regionale Konzentrationen der Zuwanderer verursacht wurde und zu großräumigen Umsiedlungsaktionen führte (Edding 1959: 36; Jolles 1965: 156), ferner die positive Beeinflussung der Altersstruktur durch die günstige Alterszusammensetzung und durch die höhere Geburtenüberschußquote der Zuwanderer (Edding 1959: 36; Nellner 1959: 102) und die durch die Abtrennung der Ostgebiete homogenisierte Agrarstruktur, die durch Großgrundbesitz und Gutsherrschaft geprägt war (Lepsius 1974).

Der Strukturwandel nach dem Zweiten Weltkrieg weist keinen gleichförmigen Entwicklungsverlauf auf. Idealtypisch werden zwei Phasen unterschieden: Zum einen die Zeit der Restauration', des Wiederaufbaus und des Wirtschaftswunders, die durch die Währungsreform 1948 und die Konstituierung der Bundesrepublik 1949 eingeleitet wurde, und zum anderen der Zeitraum seit den sechziger Jahren mit seinen tiefgreifenden qualitativen Veränderungen der wirtschaftlichen und gesellschaftlichen Struktur (Henning 1975: 186-211). Diese Periodisierungen erfolgen primär unter wirtschaftlichen Aspekten und sind typisch für die paradigmatische Betrachtung der Konsolidierung der Bun- 
desrepublik im Zuge der sich mit der Wirtschaftsentwicklung vollziehenden Wohlstandssteigerung.

Die herausragenden Dimensionen der Nachkriegsentwicklung - Wirtschaftswunder auf der einen und Millionen von Zuwanderer auf der anderen Seite - machen es notwendig, sich mit diesem auf den ersten Blick konträren Aspekt zu beschäftigen.

\subsection{Arbeitsmarkt und Arbeitslosigkeit}

In der Nachkriegszeit war der Arbeitsmarkt besonderen Belastungen ausgesetzt. Das durch Kriegsfolgen hohe Potential freigesetzter Arbeitskräfte wertstabilität und Außengleichgewicht - und somit entgegen einer Vollbeschäftigungspolitik - für die bis 1950 steigenden Arbeitslosenzahlen mitverantwortlich (Wallich 1979: 73).

Die Probleme der Aufnahme der Zuwanderer in das Beschäftigungssystem äußerten sich dementsprechend in einem sehr hohen Vertriebenenanteil von ca. $37 \%$ an der arbeitslosen Bevölkerung und wesentlich höheren altersspezifischen Arbeitslosenquoten (Schaubild 1). ${ }^{8}$

Erst 1959 entspricht der Arbeitslosenanteil der Zuwanderer ihrem Bevölkerungsanteil. Aber selbst Ende der fünfziger Jahre waren die Vertrie-

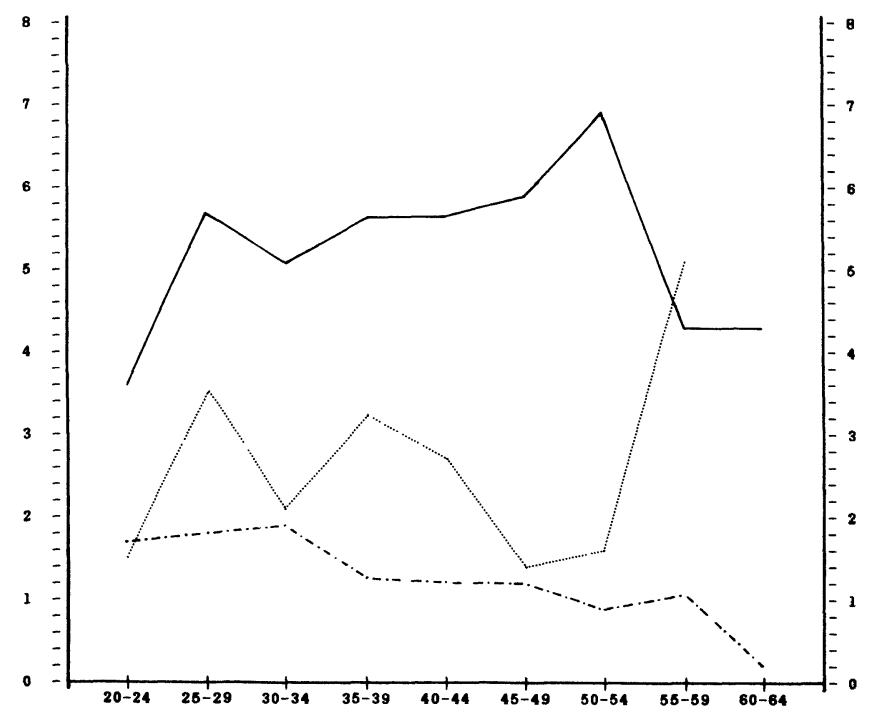

Schaubild 1

Altersspezifische Arbeitslosenquote 1950

Alter

wurde durch die Zuwanderer und Kriegsheimkehrer noch erhöht. Der Höhepunkt der Arbeitslosigkeit mit einer Arbeitslosenquote von 10,8\% lag im Jahr 1950. 1955 wurde erstmals Vollbeschäftigung erreicht. ${ }^{\text {? }}$

Weiter war die damalige wirtschaftspolitische Prioritätenfestlegung auf Preisniveaustabilität, Geld-

\footnotetext{
${ }^{7}$ Nach Definition der damaligen Bundesregierung galt bei einer Arbeitslosenquote von 5,1\% Vollbeschäftigung. 1967, nach mehreren Jahren mit einer Arbeitslosenquote von $0,4 \%$, wurde die Vollbeschäftigungsgrenze auf $0,8 \%$ Arbeitslose festgelegt, im Zuge der wirtschaftlichen Rezession jedoch bald wieder nach oben korrigiert.
}

benen von saisonal bedingter Arbeitslosigkeit (Wintermonate) aufgrund einer stärkeren Konzentration auf saisonempfindliche Berufe stärker betroffen als die einheimische Bevölkerung. Das Risiko der Arbeitslosigkeit war zudem äußerst ungleich verteilt und erfaßte bei den Zuwanderern vor allem die eigentumsgebundenen Stellungen, während sich gerade diese Gruppen bei den Einheimischen besonders resistent und stabil gegen Krisenerscheinungen zeigten. Die Arbeitslosen fanden zwar im Zuge des Wiederaufbaus rasch

\footnotetext{
${ }^{8}$ Soweit nicht anders vermerkt, handelt es sich bei den Ergebnissen um eigene Berechnungen nach der MZU 1971.
} 
eine Beschäftigung, jedoch überwiegend als unqualifizierte Arbeiter. Diese Stellung behalten sie auch im wesentlichen bei: 1971 sind $45,5 \%$ der erwerbstätigen Vertriebenen, die 1950 arbeitslos waren, als unqualifizierte Arbeiter tätig, bei den Einheimischen sind es nur $37,3 \%$.

Ein Hauptmerkmal der nach dem Zweiten Weltkrieg einsetzenden Wohlfahrtsentwicklung ist die zunehmende staatliche Absicherung von Lebensrisiken und damit die Expansion der sog. Versorgungsklasse (vgl. hierzu Lepsius 1979: 179). Neben den Arbeitslosen zählt hierzu u. a. besonders die Gruppe der Rentner. Für den Integrationsprozeß ist dies deshalb von besonderer Wichtigkeit, da selektive Benachteiligungen nicht nur in Form von Arbeitslosigkeit oder schlechten Berufschancen zu erwarten sind, sondern auch in Form von frühzeitigem Ausscheiden aus dem Erwerbsleben. 1950 ergibt sich für die zu diesem Zeitpunkt 61-73jährigen Vertriebenen eine wesentlich höhere Rentnerquote als bei den Einheimischen $(44,5 \%$ vs. $35,0 \%$ ). Selbst in der Altersgruppe der 51-60jährigen findet sich mit $10,3 \%$ bei den Vertriebenen (Einheimische 5,1\%) ein für diese Altersgruppe ungewöhnlich hoher Rentneranteil. Auch bis 1960 erfolgt keine vollständige Angleichung.

Wenn ein früher Übergang in den Ruhestand durchaus auch persönliche Präferenzen der Betroffenen entsprechen mag, so ist nicht zu übersehen, daß damit Disparitäten erzeugt werden, die über das Einkommen die Lebenslage im Alter wesentlich beeinflussen.

Da die Höhe der Bezüge nicht nur von der Höhe der Beitragszahlungen während der Erwerbstätigkeit abhängen, sondern auch von der Anzahl der Versicherungsjahre, in denen Beiträge gezahlt werden, werden vorgängige Ungleichheiten ins Alter transferiert.

Zudem bestanden viele Leistungen der gegenwärtigen Altersversorgung in der Nachkriegszeit noch nicht (die Dynamisierung der Renten erfolgte beispielsweise erst 1957) und die Lebenslage vieler Rentner war von „düsterer Armut" geprägt (Mooser 1983: 163). Trotz aller inzwischen vorgenommenen Verbesserungen der Stellung der Rentner konzentrieren sich diese 1971 immer noch auf die unteren Einkommenskategorien: 51,9\% der Vertriebenen hatten 1971 ein Einkommen bis 600 DM, wogegen nur $33,7 \%$ der Geflüchteten und $41,2 \%$ der Einheimischen zu dieser Einkommensklasse zählen. Ein weiterer Grund für die ungleiche Einkommenslage und Lebenssituation der Rentner ist sicherlich auch die unterschiedliche Verteilung von
Tabelle 1 Nettoeinkommen von Rentnern 1971

\begin{tabular}{llllr}
\hline $\begin{array}{l}\text { Einkommen } \\
\text { in DM }\end{array}$ & $0-600$ & $600-800$ & $800-1200$ & $1200+$ \\
\hline Vertriebene & 51.5 & 25.8 & 16.2 & 6.5 \\
Flüchtlinge & 34.0 & 26.4 & 24.4 & 15.1 \\
Einheimische & 40.8 & 30.2 & 21.1 & 7.9 \\
\hline Gesamt & 42.5 & 29.2 & 20.4 & 8.0 \\
\hline
\end{tabular}

Vertriebenen, Flüchtigen und Einheimischen auf verschiedene Positionen in der Erwerbsstruktur, auf die noch ausführlich einzugehen sein wird.

Frühzeitiges Ausscheiden aus dem Erwerbsleben und höhere Arbeitslosigkeit kennzeichnen ein Bild von Vertriebenen, das nicht nur in den Nachkriegsjahren von einer Konzentration auf die Versorgungsklasse geprägt ist, sondern darüber hinaus über weiterdauernde Einkommensungleichheiten in die Gegenwart transformiert wird.

Aus der Arbeitsmarkt- und Versorgungssituation der Bevölkerung wird oft der Schluß gezogen, daß die Zuwanderer eine Belastung darstellten, ohne die sich der Wiederaufbau und die wirtschaftliche Rekonstruktionsphase noch schneller vollzogen hätten (vgl. z. B. Edding 1959: 20; Jolles 1965: 221). Dabei werden jedoch zwei Dimensionen in unzulässiger Weise miteinander verknüpft. Die eine Seite ist die subjektive Lage der Zuwanderer in puncto Arbeits-, Ernährungs- und Wohnungsbereich, die sich für viele Familien in Armutssituationen und als Leben am Rande des Existenzminimums äußerte. Die andere Seite ist die ,objektive“ Auswirkung der Zuwanderung auf den Arbeitsmarkt. Durch die Zuwanderer ergab sich nicht nur eine erhöhte und kontinuierliche Nachfrage nach Verbrauchs- und Konsumgütern mit entsprechenden Rückwirkungen auf die jeweiligen Industrien, sie stellten darüberhinaus ein enormes Arbeitskräftepotential dar und beeinflußten die Entwicklung der deutschen Wirtschaft durch eine Erhöhung der verfügbaren Einsatzreserven und des westdeutschen Wachstumspotentials positiv (vgl. Abelshauser 1975: 127).

Diese Kontroverse - Belastungsfaktor versus Antriebsmotor der wirtschaftlichen Entwicklung - ist sicherlich nicht definitiv zu entscheiden. Es kann jedoch festgehalten werden, daß nach dem Krieg eine extensive Produktionsweise überwog. D. h. daß das Wirtschaftswachstum vor allem über den Einsatz zusätzlicher Arbeitskräfte erzielt wurde, was nicht zuletzt auch eine Auswirkung der $\mathrm{Zu}$ - 
wanderungen war. Um jedoch ähnliche Wachstumsraten ohne diese zuströmenden Ressourcen an menschlicher Arbeitskraft und Qualifikation zu erzielen, hätte es entweder erheblicher Umschichtungsprozesse bei der einheimischen Bevölkerung bedurft oder aber eine Verringerung ihrer Aufstiegschancen zur Folge gehabt. Auch die in diesem Zusammenhang geäußerte Hypothese einer schnelleren Umstrukturierung des Agarbereiches (ohne die Zuwanderer) mag im Grunde richtig sein. Es ist jedoch wahrscheinlicher, daß sich die wirtschaftliche Entwicklung - entsprechend dem Trend anderer westlicher Länder - langsamer vollzogen hätte, zumal sich tiefgreifende Veränderungen der Arbeitsstruktur über technische Innovationen nicht innerhalb weniger Jahre durchsetzen lassen. $^{9}$

Das Problem der Arbeitslosigkeit ist auf die fünfziger Jahre begrenzt. Von fortdauernder Bedeutung sind jedoch die sozioökonomischen Umstrukturierungen, die mit den Zuwanderungswellen einhergingen.

\subsection{Die Umschichtung der Wirtschafts- und Sozialstruktur der Bundesrepublik bis 1971}

Eine der sozialstrukturell bedeutsamsten Entwicklungen dieses Jahrhunderts ist die langfristige $\mathrm{Ab}$ nahme des primären Sektors (Landwirtschaft, Forsten, Fischerei), obgleich zwischen 1939 und 1950 die Zahl der Beschäftigten aufgrund der relativen Unversehrtheit dieses Wirtschaftszweiges anstieg.

Vertriebene, Flüchtlinge und Einheimische weisen sehr unterschiedliche Ausgangsstrukturen auf: 1939 war ein Fünftel der Vertriebenen (der MZU Stichprobe) im primären Sektor beschäftigt vs. 14,4\% der Einheimischen und nur 9,2\% der 1971 in der BRD lebenden Flüchtlinge. Dieses Bild wirft die Frage auf, inwieweit die zugewanderten Landwirte erneut eine Beschäftigung im Agrarbereich fanden. A priori standen der Eingliederung schwerwiegende Probleme entgegen, da nur wenig geeignetes Land vorhanden war, bzw. nur wenig durch Kultivierung hinzugewonnen werden konnte und die Alliierten keine Bodenreform durchführ-

${ }^{9}$ Eine noch frühere Initiierung des Gastarbeiterzustroms wäre unrealistisch gewesen. Neben den rechtlich-politischen Voraussetzungen fehlte das später vorhandene Wirtschaftswachstum, das aufgrund der besseren Verdienstmöglichkeiten (als in den Anwerbeländern) ein wesentlicher Grund für die Gastarbeiterzuwanderung war. ten. Die große Anzahl von geschaffenen Stellen für zugewanderte Landwirte (bis 1975 ca. 207 Tsd Voll- und Nebenerwerbsstellen bei annähernd 300 Tsd zugewanderten Landwirten; Korspeter/Haack 1977: 284) ist sicherlich auf den ersten Blick ein Argument für die erfolgreiche Wiedereingliederung. Dennoch erfolgte eine stetige Abnahme des Anteils der im primären Sektor Beschäftigten bei den Vertriebenen, während sich der Reduktionsprozeß bei der einheimischen Bevölkerung wesentlich langsamer vollzog. Wie unterschiedlich sich die Strukturen des primären Sektors bei Zuwanderern und Einheimischen selbst 1971 noch darstellten, zeigen zwei Beispiele.

Neben der absoluten und relativen Abnahme des primären Sektors erfolgte für die Zugewanderten entgegen dem Trend der zunehmenden Betriebskonzentration (Henning 1975: 220) - eine Verlagerung in der Betriebsgröße von überwiegend Mittelund Großbetrieben zu Kleinbetrieben. 1939 besaßen von den (vertriebenen) Landwirten noch $37,6 \%$ einen Betrieb mit über 100 ha. 1971 ist dieser Anteil nahezu Null. 1976 sind von den ca. 194 Tsd Betrieben, die durch Zuwanderer bewirtschaftet werden, nur noch $11,8 \%$ Vollerwerbsstellen mit einer Fläche von 5 ha (bei einem Gesamtanteil von $57 \%$ an landwirtschaftlichen Betrieben über 5 ha in der Bundesrepublik), 25,5\% Nebenerwerbsstellen mit 0,5-5 ha und $63 \%$ Kleinstellen mit einer Fläche unter 0,5 ha (Berechnung nach Korspeter/Haack 1977: 284).

Mit dieser Entwicklung ist die Tatsache verbunden, daß sich auf einer mehr, qualitativen' Ebene die beruflichen Stellungen innerhalb des primären Sektors bei Einheimischen und Vertriebenen unterschiedlich verlagern.

Bei den einheimischen Erwerbstätigen steigt der Anteil der selbständigen Landwirte bis 1971 - bei einem nur noch geringen Arbeiteranteil von 15,8\% und einem Anteil von 10,8\% mithelfenden Familienangehörigen - auf zwei Drittel an. Diese Entwicklung dokumentiert den zunehmenden Mechanisierungs- und Rationalisierungsproze $B$ und die Entwicklung zum reinen Familienbetrieb. Konträr dazu erhöht sich bei den Vertriebenen zwischen 1939 und 1950 die Zahl der Arbeiter auf vier Fünftel der Beschäftigten: bei $70 \mathrm{v}$. $\mathrm{H}$. in un- und angelernten Positionen und gleichzeitiger Reduktion des Anteils der selbständigen Landwirte von $16,7 \%$ (1939) auf 10,6\% (1950). Bis 1971 baut sich dieses strukturelle Ungleichgewicht keineswegs ab: nur $35,5 \%$ der Vertriebenen im primären Sektor waren zum Erhebungszeitpunkt selbständige 
Landwirte. Der Arbeiteranteil ist mit 46,6\% gegenüber den Einheimischen auch 1971 noch bedeutend größer.

Schaubild 2 zeigt, wie sich diese Reduktion und Verlagerung vollzog. Strukturänderungen können sowohl dadurch erfolgen, daß nachfolgende Generationen den Hof nicht übernehmen, als auch da-

Schaubild 2 Die Verteilung von Kohortenmitgliedern auf den Agrarsektor im Lebensverlauf, in v.H. der Erwerbspersonen dieser Kohorten

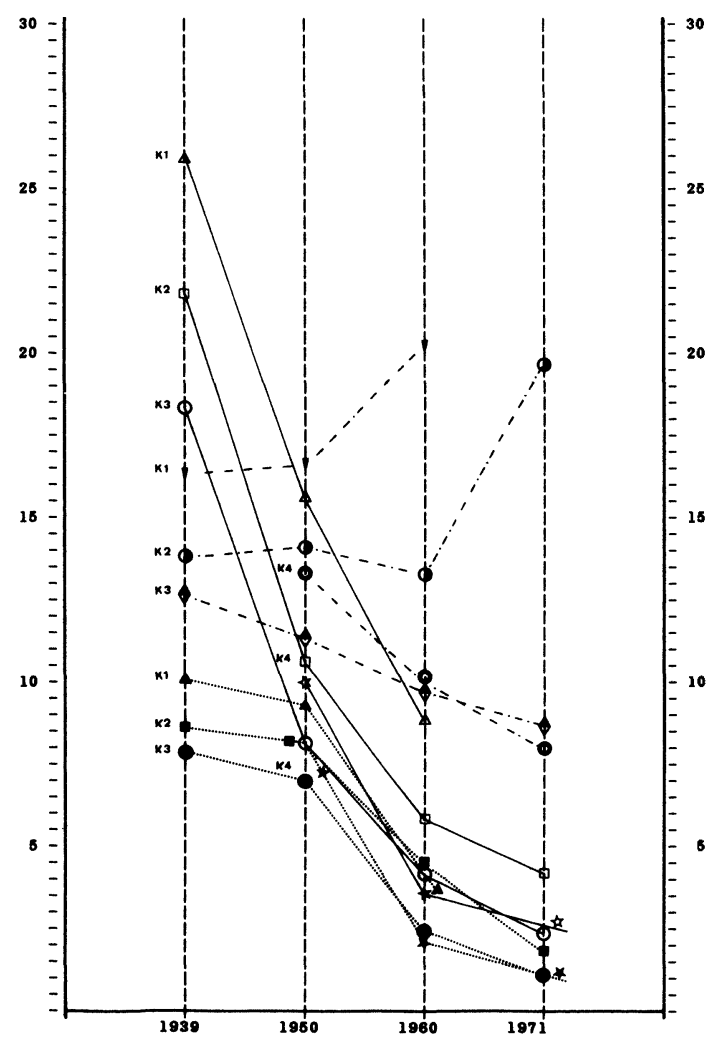

Vertriebene

Flüchtlinge

$\cdot \ldots \cdot \cdot \cdot \ldots$ Einheimische

Geburtsjahr:

Kohorte 1: $\quad 1890-1899$

Kohorte 2: $\quad 1900-1909$

Kohorte 3: $1910-1919$

Kohorte 4: $\quad 1920-1929$

durchschnittliches Alter:

1939: 45 ; 1950: 55 ; 1960: 65

1939: 35 ; 1950: 45 ; 1960: 55 ; 1971: 65

1939: 25 ; 1950: 35; 1960: 45; 1971: 55

1950: 25 ; 1960: 35 ; 1971: 45 durch, daß schon berufstätige Generationen gezwungen sind, in einen anderen Sektor zu wechseln.

Der erste Weg ist sicherlich mit geringeren psychischen Kosten verbunden, da ein erzwungener Berufswechsel gerade in diesem ,bodenverbundenen Bereich schwerwiegende Änderungen von Lebensgewohnheiten und -umständen mit sich bringt.

Die Abnahme des primären Sektors vollzieht sich bei den Einheimischen im wesentlichen in der Kohortenfolge, bei den Zuwanderern dagegen auch im Lebensverlauf der Geburtskohorten, d. h. durch eine Abwanderung in lohnabhängige Arbeitsverhältnisse. Während zwischen 1939 und 1950 die Anteile der Landwirte in den einheimischen Kohorten (1, 2 und 3) nahezu unverändert bleiben und auch bis 1960 nur geringfügig abnehmen, setzt bei den Vertriebenen bis 1950 bei allen Kohorten ein deutlicher Schrumpfungsprozeß ein, den die Flüchtlinge in der nachfolgenden Dekade bis 1960 , d. h. im Zeitraum der stärksten Fluchtbewegungen, nachvollziehen. Die bekannte Stabilität des Agrarsektors nach dem Zweiten Weltkrieg erweist sich somit als ein Strukturmerkmal der Einheimischen, während der Schrumpfungsprozeß ein Phänomen der zugewanderten Bevölkerung darstellt, der sich bis 1971 hinzieht und nur allmählich nun auch die einheimischen Erwerbstätigen des primären Sektors erfaßt. Die Dramatik des Wandels wird dabei wegen der oben angeführten internen Umschichtungsprozesse unterschätzt.

Die Stabilität des primären Sektors bei der einheimischen Bevölkerung wirft die Frage nach dem Rekrutierungsreservoir des nach dem Kriege wachsenden industriellen Sektors auf: Der sekundäre Sektor (Energie, Bergbau, Verarbeitendes Gewerbe, Hoch- und Tiefbau) beschäftigte zu allen vier Zeitpunkten $(1939,1950,1960,1971)$ sowohl absolut als auch relativ - die meisten Erwerbstätigen und hatte im Zuge des Wiederaufbaus eine überragende Bedeutung. Wie auch im primären Sektor zeigen sich unterschiedliche Allokationstendenzen. Zunächst strömt - bei Zuwanderern und Einheimischen gleichermaßen - vor allem die ins Erwerbsleben nachrückende Generation, d. h. die Kohorte der 1960 21-30jährigen (Kohorte 4 in Schaubild 3) in den expandierenden produzierenden Sektor. Wesentlich dabei ist jedoch, daß die schon für $1960 \mathrm{zu}$ beobachtende Reduktion bei den Einheimischen bei den Zuwanderern nicht stattfindet.

Das zweite in dieser Deutlichkeit überraschende Ergebnis ist die völlig konträre Entwicklung bei 
Schaubild 3 Die Verteilung von Kohortenmitgliedern aut den sekundären Sektor im Lebensverlauf, in v.H. der Erwerbspersonen dieser Kohorten

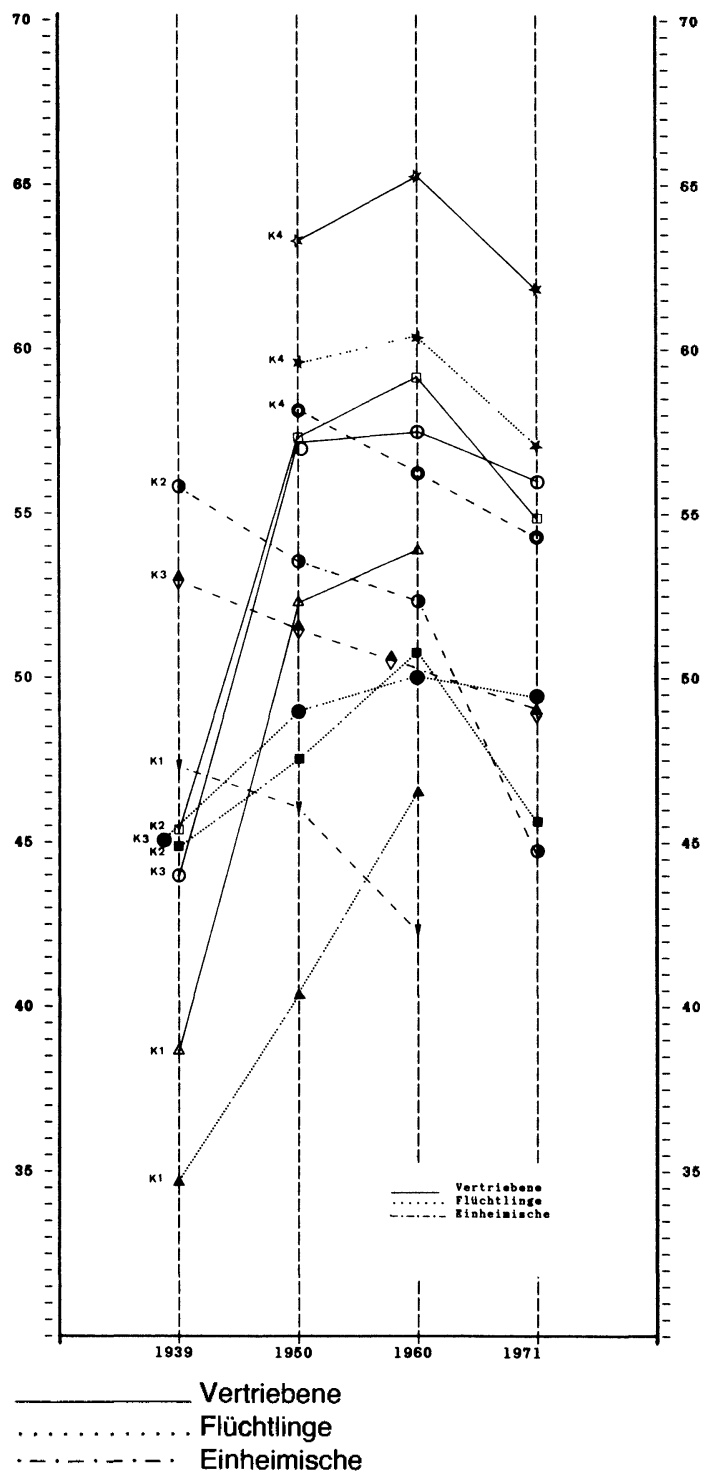

Geburtsjahr:

Kohorte 1: 1890-1899

Kohorte 2: 1900-1909

Kohorte 3: 1910-1919

Kohorte 4: $1920-1929$

durchschnittliches Alter:

1939: 45; 1950: 55 ; 1960: 65

1939: 35 ; 1950: 45 ; 1960: 55 ; 1971: 65

1939: 25 ; 1950: 35; 1960: 45; 1971: 55

1950: 25 ; 1960: 35; 1971: 45 den bereits vor dem Krieg Erwerbstätigen. Zwischen 1939 und 1950 (und in verringerter Tendenz bis 1960) steigt bei den Vertriebenen der Anteil der Arbeiter im Durchschnitt um 12 bis 15 Prozentpunkte. Dagegen zeigen die einheimischen Kohorten im gleichen Zeitraum ein anderes Verlaufsmuster. Bei ihnen nimmt die Zugehörigkeit zu diesem Sektor über alle vier Zeitpunkte hinweg bis 1971 kontinuierlich ab! ${ }^{10}$

Auf der Ebene des Beschäftigungssystems ist, in Termini von Max Webers Klassenanalyse, das Verhältnis von Besitzklassen einerseits, also Selbständigen und Produktionsmittelbesitzern, sowie Erwerbsklassen andererseits, d. h. Lohnabhängigen, von zentraler Bedeutung. Wenn auch der Schwerpunkt der Verlagerung auf die Erwerbsklassen vor 1939 anzusiedeln ist, setzt sich doch auch zwischen 1939 und 1971 diese Tendenz fort (vgl. Tabelle 2).

Tabelle 2 Selbständig vs. abhängig Beschäftigte

\begin{tabular}{llrrrr}
\hline & & 1939 & 1950 & 1960 & 1971 \\
\hline & Ver & 21.6 & 7.5 & 6.6 & 7.4 \\
Selbständige & Flü & 14.2 & 13.7 & 8.6 & 9.7 \\
(incl. Landwirte) & Ein & 19.4 & 20.0 & 18.1 & 17.4 \\
& Ges & 19.6 & 18.0 & 15.5 & 15.2 \\
\hline & Ver & 79.1 & 92.5 & 93.4 & 92.6 \\
Abhängig & Flü & 85.8 & 90.3 & 91.4 & 90.3 \\
Beschäftigte & Ein & 80.6 & 79.2 & 81.9 & 82.6 \\
& Ges & 80.4 & 82.0 & 84.5 & 84.8 \\
\hline
\end{tabular}

1971 sind ca. 85\% der erwerbstätigen Männer abhängig beschäftigt. Während jedoch die Erwerbsklasse der einheimischen Bevölkerung bis 1971 nur um 2 Prozentpunkte zunimmt, erfolgt bei den Vertriebenen eine Steigerung um 14,5 Prozentpunkte. 1971 sind über $92 \%$ der Vertriebenen in abhängigen Stellungen beschäftigt, während sich nur $82,6 \%$ der Einheimischen in entsprechenden Positionen befinden.

Der in der Erwerbsstatistik für die Bundesrepublik festgestellte Rückgang der Selbständigen ist somit vor allem ein Merkmal der zugewanderten Bevölkerung und beruht in erster Linie auf dem Rück-

${ }^{10}$ Die Entwicklung des tertiären und quartären Sektors ist für den Zeitraum bis 1960 von geringer Bedeutung, so da $B$ hier auf eine Darstellung verzichtet werden kann. Die Expansion des Dienstleistungssektors erfolgt erst nach 1960. 
gang des Anteils der Landwirte. Nach Ergebnissen der MZU 71 beträgt 1971 der Anteil der Selbständigen (ohne Landwirte) bezogen auf die Erwerbstätigen $9,8 \%$. Allerdings sind nur $6,6 \%$ der Vertriebenen als Selbständige tätig; bei den Einheimischen und Flüchtlingen dagegen jeweils ca. $10 \%$. Die positivere Entwicklung bei den Flüchtlingen ist auf zwei Faktoren zurückzuführen: Zum einen war es ihnen aufgrund der angekündigten Sozialisierungsmaßnahmen möglich, Kapital oder Produktionsmittel nach Westdeutschland zu bringen (vgl. Jolles 1965: 242). Zum anderen ist die hohe Selbständigenquote auch auf die große Zahl der Akademiker und freien Berufstätigen zurückzuführen, die weitgehend produktionsmittelunabhängig relativ geringe Erwerbsprobleme hatten und z. B. Finanzierungsprobleme über Existenzaufbaudarlehen lösen konnten.

Dagegen waren die Schwierigkeiten bei der Neugründung von Zuwandererbetrieben nicht nur durch die wirtschaftlichen Rahmenbedingungen vorprogrammiert. Die Betriebsgründungen erfolgten oft ohne Berücksichtigung der vorhandenen Infrastruktur und der gegebenen Wirtschaftsverhältnisse. Die durch die unsystematischen Standortverlagerungen hervorgerufenen Probleme (Rohstofflieferungen, Transportverhältnisse, Absatzsituation etc.) mit den besonders in Krisenzeiten entsprechenden negativen Folgen dokumentieren sich auch in der Betriebsgrößenstruktur. So hatten vor dem Krieg bei den Zuwanderern $30 \mathrm{v}$. H. einen Betrieb mit mehr als 10 Mitarbeitern. Noch deutlicher wird das Ungleichgewicht für die Vertriebenen, wenn man die Großbetriebe betrachtet. Nach dem Krieg konzentrierten sich die vertriebenen Selbständigen zunehmend auf Kleinstbetriebe (bis 1 Mitarbeiter), während Flüchtlinge bei mittleren (2-9 Mitarbeiter) und Großbetrieben überrepräsentiert sind. Diese Abnahme geht auch nach 1971 weiter. Während zu diesem Zeitpunkt noch $17 \%$ der erfaßten Betriebe in der Bundesrepublik Zuwandererbetriebe sind, reduziert sich dieser Anteil bis 1976 auf ca. 10\% (Korspeter/Haak 1979: 285).

Die zentrale Kategorie innerhalb der Erwerbsklasse ist die Arbeiterschaft. Die Entwicklung zur Wohlfahrtsgesellschaft nach dem Zweiten Weltkrieg hat im wesentlichen ihre Dimensionierung durch die soziale Lage der Arbeiter erhalten. Erst nach 1960 erfolgt eine Reduktion des zunächst durch die extensive Produktionsweise bedingten Anstiegs des absoluten und relativen Arbeiteranteils. Überraschenderweise werden jedoch die ein- heimischen Erwerbstätigen vom quantitiven Wachstum der fünfziger Jahre kaum tangiert. Dagegen verzeichnen die Vertriebenen zwischen 1939 und 1950 eine Steigerung um 20 Prozentpunkte auf 71\% der Erwerbstätigen, und auch 1971 befinden sich immer noch $58 \%$ in einer Arbeiterposition. Auch die Flüchtlinge verzeichnen einen Anstieg

Schaublld 4 Die Verteilung von Kohortenmitgliedern auf unqualifizierte Arbeiterpositionen im Lebensverlauf, in v.H. der Erwerbspersonen dieser Kohorten

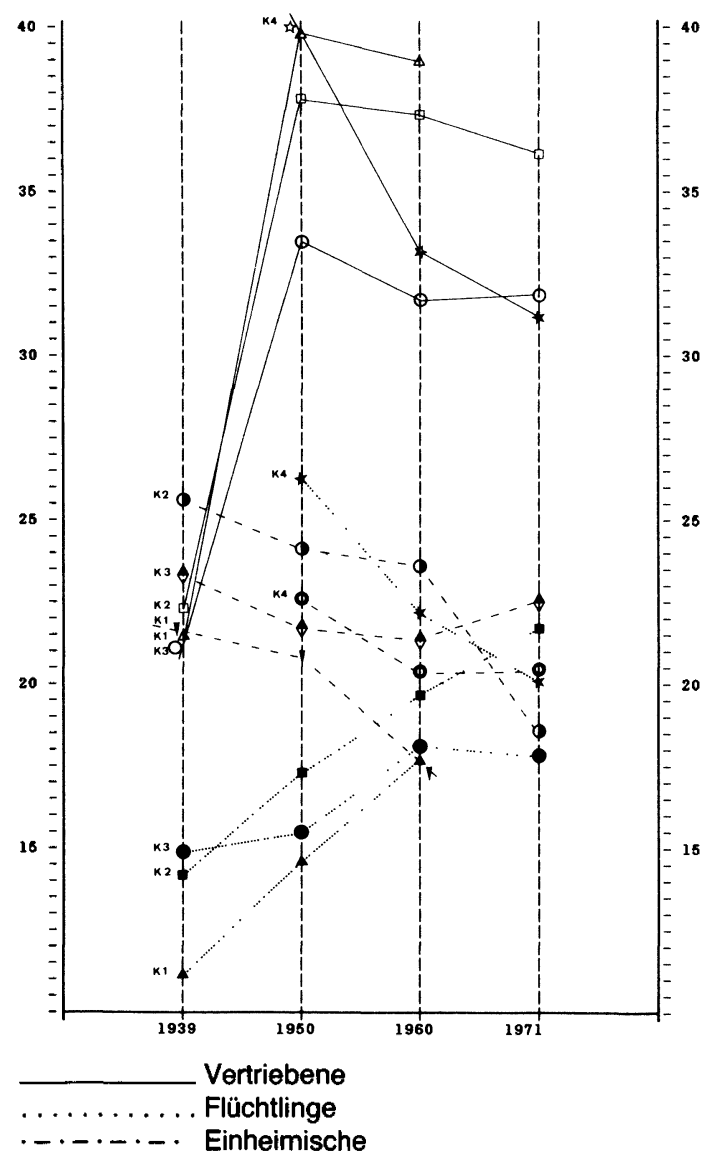

Geburtsjahr:

Kohorte 1: $1890-1899$

Kohorte 2: $1900-1909$

Kohorte 3: $1910-1919$

Kohorte 4: 1920-1929

durchschnittliches Alter:

1939: 45; 1950: 55; 1960: 65

1939: 35; 1950: 45; 1960: 55; 1971: 65

1939: 25 ; 1950: 35; 1960: 45; 1971: 55

1950: 25 ; 1960: 35; 1971: 45 
des relativen Arbeiteranteils. Sie weisen jedoch zu allen vier Zeitpunkten die niedrigsten Werte auf. $\mathrm{Da}$ besonders für die fünfziger Jahre diese Fixierung der Vertriebenen auf die Arbeiterklasse ein mitentscheidender Faktor für höhere Lebensrisiken und geringere Lebensqualität darstellt (Arbeitsplatzrisiken, Frühinvalidität etc.), braucht hier nicht explizit dargelegt zu werden (vgl. hierzu Kuczynski 1969: 212-221; Narr 1979: 245).

Auf der Kohortenebene zeigt sich eine deutliche Unterschichtung der Erwerbsstruktur, d. h. die Übertragung überwiegend unqualifizierter Tätigkeiten auf die Vertriebenen (vgl. Hoffmann-Nowotny 1973). Während sich für den Zeitpunkt der Vertreibung geringfügige Unterschiede zugunsten der Vertriebenen und deutliche Unterschiede zugunsten der Flüchtlinge ergeben, sind bis 1950 bei allen Kohorten der Vertriebenen erhebliche Steigerungen der Anteile unqualifizierter Arbeiterpositionen zu verzeichnen (vgl. Schaubild 4).

Dagegen zeigt der starke Anstieg an unqualifizierten Arbeitskräften während der Rekonstruktionsphase bei den einheimischen Kohorten fast keine Auswirkungen. Alle bereits 1939 erwerbstätigen Kohorten reduzieren im Zeitverlauf ihren Anteil an unqualifizierten Arbeiterpositionen.

Trotz einer sichtbaren Verringerung dieser ungünstigen Relation in allen Altersgruppen der Vertriebenen bis 1971 ändert sich am Übergewicht der unqualifizierten Positionen gegenüber den Einheimischen nichts. Die 1971 42-51jährigen, die nach dem Zweiten Weltkrieg und in den fünfziger Jahren erwerbstätig wurden, haben noch immer einen Anteil von $31 \%$ der Erwerbstätigen in unqualifizierten Positionen, dagegen sind es bei den gleichaltrigen Einheimischen nur 20,9\%. Auch die nachfolgende Kohorte der Vertriebenen, die zwischen 1930 und 1939 geboren wurde und zu einem Zeitpunkt den Arbeitsmarkt betrat, als bereits in zunehmendem Maße ausländische Arbeitskräfte für diese Positionen rekrutiert wurden, weist noch 1971 einen höheren Anteil an unqualifizierten Arbeiterpositionen auf.

Der für die Bundesrepublik konstatierte Wandel der Wirtschafts- und Sozialstruktur bis 1971 ist somit durch eine äußerst selektive Wirkung auf Vertriebene und in abgeschwächter Form auf Flüchtlinge gekennzeichnet. Angesichts der GröBenordnung dieser Bevölkerungsteile stellt die übliche Betrachtung des sozialen Wandels für diese Epoche ein erheblich verzerrtes „Mittelmaß“ dar, das der tatsächlichen Entwicklung eines Großteils der Bevölkerung nicht gerecht wird. Neben einer
Fixierung der Zuwanderer auf die Versorgungsklasse und der deutlichen Expansion der Arbeiterklasse ist die übliche Betrachtung des sozialen Wandels über unterschiedliche Strukturen sukzessive folgender Kohorten für die Entwicklung der Bundesrepublik nur ein Teil der zu beobachtenden „Realität“. Zwar ergeben sich z. B. für die jüngeren, d. h. neu ins Erwerbsleben eintretenden Kohorten, bei Zuwanderern und bei Einheimischen die erwarteten höheren Anteile im produzierenden Sektor - anders als bei den älteren Kohorten -, aber neben diesem "normalen" Allokationsprinzip für neu eintretende Kohorten zeigt sich für die Zuwanderer noch ein zweites Muster in der Abfolge sozialökonomischen Wandels: $\mathrm{Zu}$ einem wesentlichen Teil vollzieht sich dieser Wandel über Mobilität während des Erwerbslebens und somit als Einschnitt in der „normalen" Sequenz des Lebenslaufs.

\section{Integration und Chancengleichheit}

\subsection{Bildungssystem und Qualifikationsstruktur}

Transitorische Prozesse von Sozial- und Wirtschaftsstrukturen sind auf individueller Ebene immer mit konstanten oder veränderten beruflichen Chancenmustern verbunden, die in Abhängigkeit von der sozialen Herkunft und dem erreichten Ausbildungsniveau stehen. Wenn sich, wie oben festgestellt, sehr deutliche gruppenspezifische Wandlungsprozesse ergeben, muß die Frage nach den gruppenspezifischen Mobilitätsmustern folgen, deren Ergebnis letztlich die beobachteten Strukturänderungen darstellen. Seit Mitte der sechziger Jahre wurde in der bildungspolitischen Diskussion vor allem die Frage thematisiert, inwieweit das Bildungssystem selektive Wirkungen in der Zuordnung von Personen zu Berufspositionen hat und in welchem Ausmaß der Bildungserfolg von der sozialen Herkunft abhängt. Die Fragestellung nach dem Zusammenhang von Herkunft, Ausbildung und Beruf im Statuszuweisungsproze $B$ führte jedoch nicht zu einheitlichen Ergebnissen. (vgl. Müller/Mayer 1976: 13-21) ${ }^{11}$

Fest steht aber, daß schulische Chancen (und damit Bildungszertifikate) entscheidend für die Berufswahl (und damit auch für das Einkommen)

${ }^{11}$ Es sollte aber nicht verkannt werden, daß Bildung über die Reglementierung von Berufskarrieren hinaus hohen Stellenwert besitzt. Der Grad der Schulbildung stellt ein wesentliches Statusmerkmal dar. 
sind. Insofern können Partizipationsquoten als Indikator der Verteilung von Lebenschancen aufgefaßt werden.

Vertriebene, Flüchtlinge und Einheimische unterscheiden sich hinsichtlich ihrer Qualifikationsstruktur erheblich. Nur jeder zehnte Flüchtling hat 1971 einen Abschluß ohne Lehre, bei den Vertriebenen und Einheimischen hingegen jeder fünfte. Ähnliche Relationen finden sich bei den Abiturienten und Hochschulabsolventen, deren Anteil bei den Flüchtlingen annähernd doppelt so hoch ist wie bei Vertriebenen bzw. Einheimischen.

Diese Differenzen können als das Ergebnis zweier unterschiedlicher Prozesse betrachtet werden: Erstens als Folge selektiver Wanderungen, d. h. daß überwiegend qualifizierte Erwerbstätige mit den Lebensbedingungen in der DDR unzufrieden waren, bzw. im Westen die vergleichsweise besseren Chancen sahen; zweitens als Ergebnis von Benachteiligungen bei der Wahrnehmung von Bildungschancen infolge der Wanderung und unterschiedlichen Bildungsaspirationen der Zuwanderer, die wegen der Notsituation der Anfangsjahre eine auf schnelle Erwerbstätigkeit ausgerichtete Ausbildung der Kinder einer längeren schulischen Verweildauer vorzogen.

Der Zeitraum stärkster Belastung liegt ohne Zweifel zwischen Kriegsende und Gründung der Bundesrepublik. Die bis 1919 Geborenen waren bei Kriegsende 26 Jahre und älter. Sie hatten somit zum größten Teil ihre Ausbildung abgeschlossen. Auftretende Differenzen in der Bildungsstruktur der drei ältesten Kohorten sind deshalb auf selektive Wanderungsbewegungen zurückzuführen, bzw. auf unterschiedliche Ausgangsstrukturen in den Wanderungsgebieten. Bezüglich der Flüchtlinge erweitern sich diese Bedingungen noch auf die folgende Kohorte der zwischen 1920 und 1929 Geborenen, da der Großteil der Flüchtlinge erst nach 1950 in die Bundesrepublik kam, was eine zeitliche Verschiebung der Bruchstellen bedeutet.

Vertriebene und Einheimische zeigen bei den vier ältesten Kohorten keine wesentlichen Differenzen. Der Anteil der Volksschüler ohne Lehre nimmt bei beiden Gruppen kontinuierlich auf ca. ein Fünftel $a b$, während die mittleren und höheren Abschlüsse bei den Vertriebenen sogar etwas höher als bei den Einheimischen liegen. Deutliche Diskrepanzen ergeben sich aber bei den Flüchtlingen. Nur maximal ein Fünftel der bis 1929 Geborenen weist einen Abschluß ohne Lehre auf, während der Anteil der mittleren und höheren $\mathrm{Ab}$ schlüsse über $40 \%$ beträgt.
Diese selektive Wanderung brachte einen enormen Transfer an Humankapitel mit sich, der andernfalls nur über beträchtliche Bildungsinvestitionen der Bundesrepublik zu erreichen gewesen wäre. Die DDR verlor in den fünfziger Jahren ca. ein Drittel ihrer Akademiker und eine beträchtliche Zahl von Technikern und Facharbeitern. Ohne diese Abwanderung wäre der Hochqualifiziertenanteil in der DDR bereits 1964 höher gewesen als in der BRD. In der Bundesrepublik führte dieser Humankapitaltransfer dazu, daß die Ausgaben für den Ausbildungssektor unter den relativen Standard der Weimarer Republik fielen, ohne daß

Schaubild 5 Ausbildungsabschlüsse 1971 nach Geburtskohorten, in v. $\mathrm{H}$.

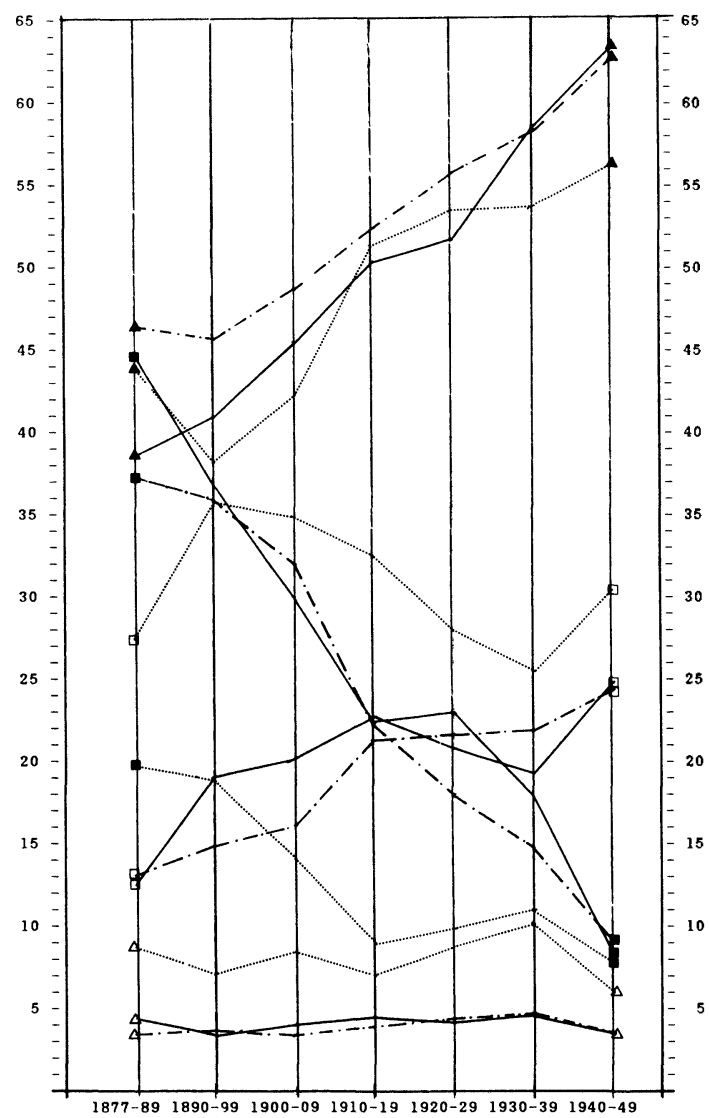

Vertriebene

Flüchtlinge

.......... Einheimische

- Volksschule ohne Lehre

- Volksschule mit Lehre

$\square \quad$ mittlere Ausbildung

$\triangle \quad$ Universität 
deswegen die Wachstumschancen der Wirtschaft verringert wurden. Den Wert dieses aus der DDR importierten Humankapitals schätzt Abelshauser (1983: 96) auf ca. 30 Milliarden DM. Das Versiegen des Zustroms dieser qualifizierten Arbeitskräfte durch die hermetische Schließung der Grenze deckte erst die Lücken in der Infrastruktur des vernachlässigten Bildungssystems der Bundesrepublik auf und war ein wesentlicher Anstoß für die Diskussion um die „Bildungskatastrophe“ in den sechziger Jahren.

Deutliche Auswirkungen der Kriegsfolgen zeigen sich nun bei den Kohorten, die zwischen 1920 und 1939 geboren wurden und deren Alter bei Kriegsende sieben bis fünfundzwanzig Jahre betrug. Während bei der einheimischen Bevölkerung der Anteil der Absolventen ohne Lehre kontinuierlich zurückgeht, wird dieser Trend bei den Vertriebenen unterbrochen, deren Anteil an unqualifizierten Abschlüssen auf $23 \%$ ansteigt.

Die auch bei den jüngeren Kohorten weiterhin relativ positive Bildungsstruktur der Flüchtlinge läßt sich nicht mehr nur mit einer selektiven Fluchtbewegung erklären. Auch ein Herkunftseffekt scheint hier wirksam zu werden. Das hohe Bildungsniveau der geflüchteten Eltern schlägt sich in einem ebenso hohen Aspirationsniveau in bezug auf die schulische und berufliche Ausbildung der Kinder nieder, zumal der Zwang zur unmittelbaren Subsistenz nicht mehr in diesem $\mathrm{Maß}$ wie in der Nachkriegszeit gegeben war. Die jüngste Kohorte der 197121 bis 31jährigen Flüchtlinge, die sich in den sechziger Jahren in Ausbildung befand, hat mit ca. $36 \%$ einen hohen Anteil an mittleren und hohen Abschlüssen, wogegen der Anteil an sekundärer und tertiärer Bildung bei den Einheimischen $(27,9 \%)$ und Vertriebenen $(28,5 \%)$ geringer ausfällt.

Insgesamt zeigt sich die über den langen Untersuchungszeitraum verbesserte Bildungssituation für alle drei Gruppen. Sie dokumentiert sich vor allem sowohl im Anstieg der Volksschulabsolventen mit Lehre als auch im Anstieg der mittleren Bildungsabschlüsse, wenn sich auch aufgrund des Alters dieser Kohorten noch keine Aussagen über evtl. Auswirkungen der Ende der sechziger Jahre einsetzenden Bildungsexpansion treffen lassen.

\subsection{Herkunftsspezifische Ausbildungschancen}

Die Feststellung kohortenspezifischer Differenzen in bezug auf die allgemeinbildenden Abschlüsse wirft weitere Fragen auf. Eine Vielzahl bildungsso- ziologischer Untersuchungen erbrachte den Nachweis, daß neben der individuellen Leistungsfähigkeit $u$. a. auch die soziale Herkunft, die regionale Herkunft, das Geschlecht, die Konfession und die Familiengröße wesentliche Determinanten des Schulerfolges darstellen, als deren Ergebnis die festgestellten Bildungs- und Ausbildungsquoten fungieren.

Für die vorliegende Problematik bleibt die Frage, inwieweit die festgestellten Differenzen bei den Bildungsquoten mit der (geographischen und sozialen) Herkunft variieren und ob sich für Zuwanderer und Einheimische die gleichen selektiven Muster herkunftsspezifischer Ausbildungschancen ergeben. ${ }^{12}$

Tabelle 3 zeigt den bivariaten Zusammenhang zwischen sozialer Herkunft und erreichtem Ausbildungsabschluß der Söhne für die 1920-1929 Geborenen, deren Ausbildungszeit in die Kriegs- und Vertreibungsphase fiel.

Für die Söhne von einheimischen Landwirten ist der Volksschulabschluß ohne Lehre dominierend, während schon mehr als die Hälfte der Söhne von einheimischen unqualifizierten Arbeitern eine gewerbliche Lehre durchläuft. Bei den Vertriebenen sind jedoch auch die Söhne von qualifizierten Arbeitern, Selbständigen und der einfachen Mittelschicht (einfache Beamte und Angestellte) deutlich von Dequalifizierungsprozessen betroffen: $12 \%$ bis $14 \%$ erreichen nur einen Abschluß ohne Lehre.

Bei den Söhnen der zugewanderten Landwirte allerdings zeigt sich im Vergleich zu den Einheimischen ein geringerer Anteil mit unqualifizierten Abschlüssen. Dies dürfte seine Ursache darin haben, daß bei einer Hofübernahme durch den Sohn dieser in der Regel keine fachspezifische Lehre absolviert hatte. Für die zugewanderten Landwirte, die - bedingt durch die Wanderung - nicht mehr die Chance der Fortführung eines eigenen landwirtschaftlichen Betriebes durch die Nachkommen sahen - sei es durch Betriebsverlust oder

${ }^{12}$ Die Erweiterung um den dynamischen Aspekt der Zugangschancen zu Bildung bzw. beruflicher Stellung ist notwendig, da sich über ungleiche Verteilungen keine Aussagen über den Grad der Chancengleichheit treffen lassen. Im Gegensatz zu Rekrutierungsquoten (,inflow') sind Chancen nicht als Anteile an einer Gesamtzahl von Absolventen oder Abschlüssen zu bestimmen, sondern als Anteil an der Gesamtzahl von Kindern mit gegebener sozialer Herkunft, d. h. als Abstromprozente (,outflow'). 
Tabelle 3 Beruf der Väter - Ausbildung der Sǒhne, in v. H., 1920-1929 geborene Männer

\begin{tabular}{|c|c|c|c|c|c|c|c|}
\hline \multirow[t]{2}{*}{ Beruf Väter } & & \multicolumn{6}{|c|}{ Ausbildung Söhne } \\
\hline & & $\begin{array}{l}\text { Volksschule } \\
\text { ohne } \\
\text { Abschluß, } \\
\text { ohne Lehre }\end{array}$ & $\begin{array}{l}\text { Volksschule } \\
\text { + gewerbl. } \\
\text { Lehre }\end{array}$ & $\begin{array}{l}\text { Volksschule } \\
\text { + kaufm. } \\
\text { Lehre }\end{array}$ & $\begin{array}{l}\text { mittlere } \\
\text { Reife }\end{array}$ & $\begin{array}{l}\text { Abitur, } \\
\text { Ingenieur- } \\
\text { schule }\end{array}$ & $\begin{array}{l}\text { Uni- } \\
\text { ver- } \\
\text { sität }\end{array}$ \\
\hline Selbständige & $\begin{array}{l}\text { Ver } \\
\text { Flü } \\
\text { Ein }\end{array}$ & $\begin{array}{r}12.8 \\
4.8 \\
7.6\end{array}$ & $\begin{array}{l}41.5 \\
32.8 \\
40.2\end{array}$ & $\begin{array}{r}8.7 \\
6.6 \\
10.5\end{array}$ & $\begin{array}{l}21.7 \\
26.6 \\
26.6\end{array}$ & $\begin{array}{r}9.3 \\
15.7 \\
8.6\end{array}$ & $\begin{array}{r}6.0 \\
13.5 \\
16.9\end{array}$ \\
\hline Freie Berufe & $\begin{array}{l}\text { Ver } \\
\text { Flü } \\
\text { Ein }\end{array}$ & $\begin{array}{l}- \\
12.5^{\star} \\
-\end{array}$ & $\begin{array}{l}- \\
\overline{9} .3\end{array}$ & $\begin{array}{l}6.7^{\star} \\
- \\
-\end{array}$ & $\begin{array}{c}6.7^{*} \\
- \\
18.7\end{array}$ & $\begin{array}{l}6.7^{\star} \\
25.0 \\
13.3\end{array}$ & $\begin{array}{l}80.0 \\
62.5^{*} \\
58.7\end{array}$ \\
\hline $\begin{array}{l}\text { gehobene } \\
\text { Mittelschicht }\end{array}$ & $\begin{array}{l}\text { Ver } \\
\text { Flü } \\
\text { Ein }\end{array}$ & $\begin{array}{l}2.3^{*} \\
3.2^{*} \\
1.7\end{array}$ & $\begin{array}{r}11.3 \\
7.4 \\
8.8\end{array}$ & $\begin{array}{l}7.2 \\
4.2^{\star} \\
5.2\end{array}$ & $\begin{array}{l}26.3 \\
22.6 \\
28.9\end{array}$ & $\begin{array}{l}21.7 \\
23.2 \\
24.1\end{array}$ & $\begin{array}{l}31.2 \\
39.5 \\
31.3\end{array}$ \\
\hline $\begin{array}{l}\text { mittlere } \\
\text { Mittelschicht }\end{array}$ & $\begin{array}{l}\text { Ver } \\
\text { Flü } \\
\text { Ein }\end{array}$ & $\begin{array}{l}7.6 \\
3.2^{\star} \\
3.5\end{array}$ & $\begin{array}{l}26.3 \\
30.1 \\
29.3\end{array}$ & $\begin{array}{r}11.4 \\
9.7 \\
14.9\end{array}$ & $\begin{array}{l}35.5 \\
28.0 \\
30.4\end{array}$ & $\begin{array}{l}13.3 \\
17.2 \\
13.6\end{array}$ & $\begin{array}{r}5.9 \\
11.8 \\
8.3\end{array}$ \\
\hline $\begin{array}{l}\text { einfache } \\
\text { Mittelschicht }\end{array}$ & $\begin{array}{l}\text { Ver } \\
\text { Flü } \\
\text { Ein }\end{array}$ & $\begin{array}{c}13.6 \\
3.6^{\star} \\
7.7\end{array}$ & $\begin{array}{l}45.1 \\
40.5 \\
47.2\end{array}$ & $\begin{array}{l}13.6 \\
13.1 \\
15.5\end{array}$ & $\begin{array}{l}21.1 \\
25.0 \\
20.6\end{array}$ & $\begin{array}{r}3.9 \\
13.1 \\
6.5\end{array}$ & $\begin{array}{l}2.6 \\
4.8 \\
2.5\end{array}$ \\
\hline Arbeiterelite & $\begin{array}{l}\text { Ver } \\
\text { Flü } \\
\text { Ein }\end{array}$ & $\begin{array}{l}8.2 \\
2.1^{\star} \\
6.1^{4}\end{array}$ & $\begin{array}{l}48.0 \\
46.9 \\
48.6\end{array}$ & $\begin{array}{l}10.0 \\
13.5 \\
14.5\end{array}$ & $\begin{array}{l}23.3 \\
25.0 \\
21.8\end{array}$ & $\begin{array}{r}5.4 \\
10.4 \\
5.8\end{array}$ & $\begin{array}{l}5.0 \\
2.1^{*} \\
3.2\end{array}$ \\
\hline $\begin{array}{l}\text { gelernte } \\
\text { Arbeiter }\end{array}$ & $\begin{array}{l}\text { Ver } \\
\text { Flü } \\
\text { Ein }\end{array}$ & $\begin{array}{r}15.3 \\
6.6 \\
9.2\end{array}$ & $\begin{array}{l}61.7 \\
65.3 \\
64.7\end{array}$ & $\begin{array}{r}7.5 \\
7.4 \\
10.5\end{array}$ & $\begin{array}{l}13.0 \\
15.2 \\
12.2\end{array}$ & $\begin{array}{l}1.8 \\
3.4 \\
2.5\end{array}$ & $\begin{array}{l}0.8^{*} \\
2.0^{\star} \\
0.9\end{array}$ \\
\hline $\begin{array}{l}\text { un-, angelernte } \\
\text { Arbeiter }\end{array}$ & $\begin{array}{l}\text { Ver } \\
\text { Flü } \\
\text { Ein }\end{array}$ & $\begin{array}{l}36.5 \\
21.7 \\
26.7\end{array}$ & $\begin{array}{l}51.5 \\
62.8 \\
58.2\end{array}$ & $\begin{array}{l}5.3 \\
7.5 \\
7.2\end{array}$ & $\begin{array}{l}5.4 \\
5.9 \\
6.8\end{array}$ & $\begin{array}{l}1.0 \\
2.0^{*} \\
0.8\end{array}$ & $\begin{array}{l}0.2^{*} \\
\overline{0.3}\end{array}$ \\
\hline Landwirte & $\begin{array}{l}\text { Ver } \\
\text { Flü } \\
\text { Ein }\end{array}$ & $\begin{array}{l}40.8 \\
26.7 \\
49.6\end{array}$ & $\begin{array}{l}39.4 \\
41.7 \\
31.5\end{array}$ & $\begin{array}{l}3.7 \\
6.7^{\star} \\
3.2\end{array}$ & $\begin{array}{l}12.7 \\
19.2 \\
13.3\end{array}$ & $\begin{array}{l}1.5 \\
4.2^{*} \\
1.5\end{array}$ & $\begin{array}{l}1.9 \\
1.7^{*} \\
0.9\end{array}$ \\
\hline & Gesamt & 13.9 & 49.7 & 8.2 & 18.3 & 5.2 & 4.8 \\
\hline
\end{tabular}

$*: N \leq 10$

Verlagerung auf $\mathrm{Zu}$ - und Nebenerwerbsbetriebe -, war ein anderes Bildungsverhalten der Söhne angemessen.

Der überwiegende Abschluß ist bei allen Gruppen, mit Ausnahme der freien Berufe und der gehobenen Mittelschicht der Lehrabschluß. Mit der Zunahme des beruflichen Status geht der bekannte Effekt des Anstiegs qualifizierter Abschlüsse einher: Während das Bildungsverhalten einfacher
Angestellter und Beamter noch dem der qualifizierten Arbeiter ähnelt - ein Unterschied ergibt sich nur in der stärkeren Präferierung kaufmännischer Lehren bei der einfachen Mittelschicht steigt der Anteil an Abiturienten und Hochschulabsolventen bei den höheren Berufsgruppen deutlich an, mit nur noch minimalen und kaum mehr interpretierbaren Differenzen zwischen Zuwanderern und Einheimischen. 
Die Unterschiede in den Bildungschancen - mit einem deutlichen Gefälle innerhalb der Zuwanderer - sind somit vor allem ein Effekt der sozialen Herkunft. Die Unterschiede zwischen Zuwanderern und Ansässigen bei gleicher sozialer Herkunft sind geringer als die Unterschiede innerhalb der Gruppe der Vertriebenen, Flüchtlinge oder Einheimischen bei unterschiedlicher sozialer Herkunft. Die Söhne der Zuwanderer aus der einfachen Mittelschicht ähneln in puncto Bildungschancen eher den Söhnen der einheimischen Mittelschicht als den Söhnen anderer Zuwanderergruppen. D. h. weniger die Herkunft (im geographischen Sinn) als vielmehr die Schichtzugehörigkeit bestimmt in erheblichem $\mathrm{Maß}$ die Bildungsmöglichkeiten.

Ein Vergleich mit den jüngeren Kohorten (von 1930 bis 1939 und von 1940 bis 1949 Geborenen), deren Alter 1971 zwischen 32 und 41 bzw. 22 und 31 Jahren betrug, zeigt deutliche Nivellierungstendenzen (ohne Tabelle). Auch hier findet sich der Trend zur besseren Qualifikation (Lehrabschluß) sowohl bei den Söhnen vertriebener Landwirte als nun auch bei den Söhnen einheimischer Landwirte. Ebenfalls bei den Einheimischen verliert der Agrarsektor an Bedeutung und bewirkt ein anderes Bildungsverhalten für die Kinder, da schon ein qualifizierter Arbeiterberuf nur über eine Lehre zu erreichen ist. Auch die Söhne von vertriebenen unqualifizierten Arbeitern zeigen nun eine Anpassung an die Abschlüsse der Einheimischen und Flüchtlinge.

\subsection{Bildung und Zuweisung sozialer Positionen}

Nach der Feststellung eines stringenten Zusammenhangs zwischen sozialer Herkunft und schulischer Ausbildung erhebt sich die Frage nach dem Muster der Übergänge zwischen Ausbildung und Beruf: Inwieweit unterliegen Zuwanderer und Einheimische mit gleichen formalen Ausbildungsvoraussetzungen unterschiedlichen Selektionsprozessen zum Beschäftigungssystem? (Hierzu Tabelle 4)

Tabelle 4 Ausbildung - berufliche Stellung 1971, in v. H., 1920-1929 geborene Mănner

\begin{tabular}{|c|c|c|c|c|c|c|c|c|c|c|c|}
\hline \multirow[t]{2}{*}{ Ausbildung } & & \multicolumn{10}{|c|}{ Berufliche Stellung } \\
\hline & & $\begin{array}{l}\text { Selb- } \\
\text { ständige }\end{array}$ & $\begin{array}{l}\text { Freie } \\
\text { Berule }\end{array}$ & $\begin{array}{l}\text { gehobene } \\
\text { Mittel- } \\
\text { schicht }\end{array}$ & $\begin{array}{l}\text { mittlere } \\
\text { Mittel- } \\
\text { schicht }\end{array}$ & $\begin{array}{l}\text { einfache } \\
\text { Mittel- } \\
\text { schicht }\end{array}$ & $\begin{array}{l}\text { Arbeiter- } \\
\text { elite }\end{array}$ & $\begin{array}{l}\text { gelernte } \\
\text { Arbeiter }\end{array}$ & $\begin{array}{l}\text { unge- } \\
\text { lernte } \\
\text { Arbeiter }\end{array}$ & $\begin{array}{l}\text { Land- } \\
\text { wirte }\end{array}$ & Gesamt \\
\hline $\begin{array}{l}\text { Volksschule } \\
\text { ohne Abschluß, } \\
\text { ohne Lehre }\end{array}$ & $\begin{array}{l}\text { Ver } \\
\text { Flü } \\
\text { Ein }\end{array}$ & $\begin{array}{l}2.5 \\
2.8 \\
3.9\end{array}$ & $\begin{array}{l}- \\
- \\
-\end{array}$ & $\begin{array}{l}0.5^{\star} \\
2.8^{\star} \\
0.7\end{array}$ & $\begin{array}{l}2.9 \\
6.2 \\
3.5\end{array}$ & $\begin{array}{l}5.0 \\
4.5^{\star} \\
5.0\end{array}$ & $\begin{array}{l}3.5 \\
2.2^{\star} \\
2.3\end{array}$ & $\begin{array}{l}8.0 \\
9.0 \\
5.2\end{array}$ & $\begin{array}{l}75.3 \\
81.3 \\
58.7\end{array}$ & $\begin{array}{c}2.3 \\
1.1^{\star} \\
20.7\end{array}$ & $\begin{array}{l}23.0 \\
10.0 \\
18.0\end{array}$ \\
\hline $\begin{array}{l}\text { Volksschule } \\
\text { gewerbliche } \\
\text { Lehre }\end{array}$ & $\begin{array}{l}\text { Ver } \\
\text { Flü } \\
\text { Ein }\end{array}$ & $\begin{array}{r}5.2 \\
7.7 \\
11.0\end{array}$ & $\begin{array}{l}- \\
- \\
-\end{array}$ & $\begin{array}{l}2.0 \\
3.8 \\
3.3\end{array}$ & $\begin{array}{l}11.8 \\
13.4 \\
13.0\end{array}$ & $\begin{array}{l}5.6 \\
3.5 \\
4.8\end{array}$ & $\begin{array}{l}6.7 \\
6.5 \\
7.0\end{array}$ & $\begin{array}{l}43.5 \\
43.9 \\
41.5\end{array}$ & $\begin{array}{l}24.1 \\
21.0 \\
16.3\end{array}$ & $\begin{array}{l}1.1 \\
0.2^{*} \\
3.1\end{array}$ & $\begin{array}{l}44.7 \\
45.0 \\
46.9\end{array}$ \\
\hline $\begin{array}{l}\text { Volksschule } \\
\text { kaufmännische } \\
\text { Lehre }\end{array}$ & $\begin{array}{l}\text { Ver } \\
\text { Flü } \\
\text { Ein }\end{array}$ & $\begin{array}{r}9.2 \\
11.0 \\
12.1\end{array}$ & $\begin{array}{l}- \\
- \\
-\end{array}$ & $\begin{array}{l}17.5 \\
18.1 \\
23.1\end{array}$ & $\begin{array}{l}42.5 \\
38.1 \\
43.0\end{array}$ & $\begin{array}{r}11.6 \\
9.7 \\
11.5\end{array}$ & $\begin{array}{l}1.8^{\star} \\
1.9^{\star} \\
1.3\end{array}$ & $\begin{array}{l}6.6 \\
6.5 \\
2.7\end{array}$ & $\begin{array}{r}10.7 \\
14.2 \\
5.3\end{array}$ & $\begin{array}{l}- \\
0.6^{\star} \\
1.1\end{array}$ & $\begin{array}{l}7.1 \\
8.2 \\
9.0\end{array}$ \\
\hline $\begin{array}{l}\text { mittlere } \\
\text { Ausbildung }\end{array}$ & $\begin{array}{l}\text { Ver } \\
\text { Flü } \\
\text { Ein }\end{array}$ & $\begin{array}{l}11.2 \\
14.9 \\
22.1\end{array}$ & $\begin{array}{l}- \\
- \\
-\end{array}$ & $\begin{array}{l}29.6 \\
35.9 \\
31.6\end{array}$ & $\begin{array}{l}33.5 \\
29.0 \\
26.7\end{array}$ & $\begin{array}{l}5.1 \\
3.7 \\
3.7\end{array}$ & $\begin{array}{l}5.2 \\
3.4 \\
3.7\end{array}$ & $\begin{array}{l}6.8 \\
7.8 \\
3.6\end{array}$ & $\begin{array}{l}8.1 \\
4.9 \\
2.7\end{array}$ & $\begin{array}{l}0.7^{\star} \\
0.3^{\star} \\
5.9\end{array}$ & $\begin{array}{l}16.0 \\
18.4 \\
16.2\end{array}$ \\
\hline $\begin{array}{l}\text { Fach-, } \\
\text { Ingenieur- } \\
\text { schulen }\end{array}$ & $\begin{array}{l}\text { Ver } \\
\text { Flü } \\
\text { Ein }\end{array}$ & $\begin{array}{l}16.0 \\
13.4 \\
22.0\end{array}$ & $\begin{array}{l}- \\
- \\
-\end{array}$ & $\begin{array}{l}59.9 \\
64.8 \\
63.6\end{array}$ & $\begin{array}{l}19.4 \\
16.2 \\
11.1\end{array}$ & $\begin{array}{l}1.2^{\star} \\
2.2^{\star} \\
0.8\end{array}$ & $\begin{array}{l}0.9^{\star} \\
1.1^{\star} \\
0.6^{\star}\end{array}$ & $\begin{array}{l}0.9^{\star} \\
1.1^{\star} \\
0.2^{\star}\end{array}$ & $\begin{array}{l}1.2^{*} \\
1.1^{\star} \\
0.2^{\star}\end{array}$ & $\begin{array}{l}0.3^{*} \\
- \\
1.4\end{array}$ & $\begin{array}{l}4.9 \\
9.6 \\
5.4\end{array}$ \\
\hline Universität & $\begin{array}{l}\text { Ver } \\
\text { Flü } \\
\text { Ein }\end{array}$ & $\begin{array}{l}2.2 \\
2.9 \\
5.3\end{array}$ & $\begin{array}{l}20.4 \\
19.4 \\
19.3\end{array}$ & $\begin{array}{l}73.8 \\
74.1 \\
72.4\end{array}$ & $\begin{array}{l}2.9 \\
2.9 \\
2.2\end{array}$ & $\begin{array}{l}0.4^{\star} \\
- \\
0.2^{\star}\end{array}$ & $\begin{array}{l}0.4^{\star} \\
- \\
0.2^{\star}\end{array}$ & $\begin{array}{l}- \\
- \\
-\end{array}$ & $\begin{array}{l}- \\
0.6^{*} \\
-\end{array}$ & $\begin{array}{l}- \\
- \\
0.4^{*}\end{array}$ & $\begin{array}{l}4.2 \\
8.8 \\
4.4\end{array}$ \\
\hline
\end{tabular}


Wie erwartet macht sich der restringierende Faktor von Eigentum vor allem in den Zugangschancen zu den Selbständigenpositionen bemerkbar. Während 1971 zwischen $12 \%$ und $13 \%$ der älteren Einheimischen mit Lehrabschluß als Selbständige tätig sind (Handwerk und Kleingewerbe), werden nur maximal $8,5 \%$ der gleichaltrigen Vertriebenen mit Lehrabschluß Selbständige. Bei den jüngeren Kohorten verringern sich sowohl die Anteile als auch die Differenzen zwischen Zuwanderern und Einheimischen. Allerdings rühren die niedrigen Anteile auch daher, daß noch im höheren Alter Übergänge in die Selbständigkeit stattfinden. Insofern ist $\mathrm{zu}$ vermuten, daß sich die Schere im $\mathrm{Zu}$ gang zur Selbständigkeit für die jüngeren Kohorten in den siebziger und achziger Jahren mit dem Älterwerden wieder öffnet. Das hieße, daß sich die strukturellen Differenzen anhand der Vererbung von Eigentum in der Generationenfolge vom Vater zum Sohn erneut zeigen. Neben den geringeren Chancen selbständig zu werden, finden sich 1971 bei den Zuwanderern hohe Anteile an Erwerbstätigen, die trotz einer gewerblichen oder kaufmännischen Lehre in unqualifizierten Arbeiterpositionen tätig sind. Atypische Allokationsmuster zeigen sich vor allem bei den Zuwanderern mit einer kaufmännischen Lehre: $22,6 \%$ der Flüchtlinge und $19,1 \%$ der Vertriebenen befinden sich in einer für diese berufliche Qualifikationsstufe keineswegs normalen Arbeiterposition. Die Nachteile der zum Wanderungszeitpunkt bereits erwerbstätigen Vertriebenen waren somit für einen erheblichen Teil nicht nur von temporärer Natur, sondern dauerten bis zum Befragungszeitpunkt an. Da es sich hierbei um Volksschulabsolventen mit abgeschlossener Lehre handelt, kann nicht mehr argumentiert werden, daß aufgrund einer fehlenden qualifizierten Ausbildung a priori nur der Weg in eine unqualifizierte Position vorgeschrieben war. Vertriebene und in etwas geringerem Umfang auch Flüchtlinge weisen deutliche Dequalifizierungsprozesse auf, betrachtet man die Werte der einheimischen $\mathrm{Be}$ völkerung als „Normalwerte“. Die bei der Analyse der sozioökonomischen Gliederung vorgefundene unterschiedliche Stärke in der Konzentration auf Arbeiterpositionen ist somit auch als Ergebnis unterschiedlicher Allokationsmuster trotz gleicher Voraussetzungen in der Qualifikation zu sehen und nicht nur als Folge einer unterschiedlichen Qualifikationsstruktur des Arbeitskräfteangebots der Zuwanderer, also als Überhang an unqualifizierten Arbeitskräften.

Auch bei den höheren Bildungsabschlüssen ergeben sich - neben den auch hier geringeren Chan- cen in selbständige Positionen zu gelangen - ungleiche Berufschancen. Ein Fünftel der Vertriebenen mit mittlerer Ausbildung gelangt in eine Arbeiterposition, wogegen es bei den Einheimischen nur zehn von Hundert sind, die 1971 eine ihrer Ausbildung nicht entsprechende berufliche Stellung einnehmen. Die geringen Quoten bezüglich der hohen Beamten- und Angestelltenpositionen, die bei den älteren Kohorten mit Differenzen bis zu 11,5 Prozentpunkten zwischen Vertriebenen und Einheimischen noch deutlicher als bei den jüngeren Kohorten ausfallen, weisen auf eine Bruchstelle im Karriereverlauf hin. Unter der Prämisse, daß hohe Positionen für Erwerbstätige mit mittlerem Abschluß nur über sich allmählich vollziehende Berufskarrieren, wie z. B. über lange Betriebszugehörigkeit, erreicht wurden (zumindest zum damaligen Zeitpunkt), scheinen gerade die älteren Kohorten durch die Vertreibung und die lange Zeit schwierige Arbeitsmarktlage an einem solchen sukzessiven Aufstieg gehindert worden zu sein. Die relativ wenigen hohen Positionen waren den einheimischen Erwerbstätigen vorbehalten, die als Angehörige der Kernbelegschaft die Chancen der vorhandenen Aufstiegskanäle besser nutzen konnten.

In der hochqualifizierten Gruppe der Abiturienten, Fach- und Hochschulabsolventen zeigen sich die geringsten Differenzen zwischen Zuwanderern und Einheimischen. Zudem lassen sich zumindest bei den Hochschulabsolventen Kompensationen in den Differenzen zwischen Selbständigen einerseits und hohen Beamten und Angestellten andererseits treffen: Geringer besetzte Selbständigenkategorien und freie Berufe korrespondieren eindeutig mit entsprechend höher besetzten hohen Beamtenund Angestelltenkategorien. Die über das Ausbildungssystem vermittelten ungleichen Berufschancen präsentieren sich somit vor allem für die Vertriebenenbevölkerung in den unteren Ausbildungspositionen. Für die Vertriebenen werden mit zunehmendem Alter die Zugangschancen zu gleichwertigen Berufen merklich schlechter. Eine Benachteiligung ergibt sich nicht nur für die Kohorten, die in der Kriegs- und Nachkriegszeit die Ausbildungsphase durchliefen, sondern ebenfalls für die Kohorten, die zu Kriegsende bereits erwerbstätig waren. Trotz gleicher Ausbildungsabschlüsse waren sie im Wettbewerb um adäquate Berufspositionen offensichtlich entscheidend benachteiligt. Die erfahrenen Diskriminierungen wurden nicht in einer späteren Lebensphase kompensiert, sondern erweisen sich als strukturierendes Merkmal der Lebensgeschichte. 


\section{Zusammenfassung}

Der Ausgangspunkt dieser Arbeit war die Frage nach den Auswirkungen der Zuwanderungen für die Lebenschancen der einheimischen und der zugezogenen Bevölkerung, und nach den Folgen für den gesamtgesellschaftlichen Wandel. Besondere Betrachtung fanden die schulischen und beruflichen Chancen, die als determinierend für die Lebenssituation betrachtet werden können.

Der Arbeit lagen zwei konkurrierende theoretische Annahmen über den Integrationsverlauf zugrunde: Der Sichtweise eines erfolgreichen Integrationsprozesses, der bereits Mitte der fünfziger Jahre im großen Ganzen als beendet gilt, stand die Alternativ-Hypothese einer relativen Deprivierung und Benachteiligung gegenüber, deren Folgen sich auch noch 1971 als Strukturmerkmal der Zuwanderer nachweisen lassen.

Die Ergebnisse dieser Studie zeigen für die älteren Kohorten keineswegs eine vollständige Integration in der Gegenwart. Die erfolgreiche Eingliederung wird erst bei den Kindern- und Kindeskindern der Zuwanderer in vollem Umfang erreicht. Geringere schulische und berufliche Chancen und ein selektiver Strukturwandel vermitteln das Bild einer teilweise ,segregierten" gesellschaftlichen Entwicklung. Es zeigt sich, da $\beta$ aus sozialhistorischer Perspektive die ersten Dekaden nach dem Zweiten Weltkrieg noch viele Fragen offen lassen und der Integrationsproze $\beta$ einer differenzierteren $\mathrm{Be}$ trachtung bedarf. Es wurde aber auch deutlich, daß es nicht die Vertriebenen oder die Flüchtlinge als homogene gesellschaftliche Einheiten gibt, sondern daß sich innerhalb der Zuwanderer vielfältige Differenzierungslinien ergeben, die ebenfalls unterschiedliche Integrationsverläufe implizieren.

\section{Literatur}

Abelshauser, W., 1975: Wirtschaft in Westdeutschland 1945-1948. Rekonstruktion und Wachstumsbedingungen in der amerikanischen und britischen Zone, Stuttgart.

Abelshauser, W., 1983: Wirtschaftsgeschichte der Bundesrepublik Deutschland 1945-1980, Frankfurt.

Becker, J., Stammen, T., Waldemann, P. (Hrsg.), 1979: Vorgeschichte der Bundesrepublik Deutschland. Zwischen Kapitulation und Grundgesetz, München.

Bethlehem, S., 1982: Heimatvertreibung, DDR-Flucht, Gastarbeiterwanderung. Wanderungsströme und Wanderungspolitik in der Bundesrepublik Deutschland, Stuttgart: Clett-Cotta.

Dahrendorf, R., 1977: Gesellschaft und Demokratie in Deutschland, München: dtv
Edding, F., 1959: Bevölkerung und Wirtschaft, in: Lemberg/Edding 1959, Band II, S. 1-53.

Grosser, A., 1982: Geschichte Deutschlands seit 1945. Eine Bilanz, München: dtv.

Henning, F. W., 1975: Das industrialisierte Deutschland 1914-1972, Paderborn: UTB.

Hoffmann-Nowotny, H. J., 1973: Soziologie des Fremdarbeiterproblems. Eine theoretische und empirische Analyse am Beispiel der Schweiz, Stuttgart.

Hoffmann-Nowotny, H. J./Hondrich, K. O., 1981: Ausländer in der Bundesrepublik Deutschland und in der Schweiz. Segregation und Integration: eine vergleichende Untersuchung, Frankfurt: Campus.

Jolles, H., 1965: Zur Soziologie der Heimatvertriebenen und Flüchtlinge, Köln, Berlin.

Korspeter, L., Haack, W., 1977: Politik für Vertriebene, Flüchtlinge, Heimkehrer, politische Häftlinge und Aussiedler, in: Bartolomäi et al.: Sozialpolitik nach 1945. Geschichte und Analysen, Bonn-Bad Godesberg, Köln, S. 275-295

Kuczynski, J., 1969: So war es wirklich. Ein Rückblick auf 20 Jahre Bundesrepublik, Berlin.

Lemberg, E., Edding, F. (Hrsg.), 1959: Die Vertriebenen in Westdeutschland. Ihre Eingliederung und ihr Einfluß auf Gesellschaft, Politik und Geistesleben. Drei Bände, Kiel: Ferdinand Hirt.

Lepsius, M. R., 1974: Sozialstruktur und soziale Schichtung in der Bundesrepublik Deutschland, in: Löwenthal, R., Schwarz, H. P. (Hrsg.), Die zweite Republik. 25 Jahre Bundesrepublik Deutschland - Eine Bilanz, Stuttgart-Degerloch: Seewald.

Lepsius, M. R., 1979: Soziale Ungleichheit und Klassenstrukturen in der Bundesrepublik Deutschland, in: Wehler, H. U. (Hrsg.), Klassen in der europäischen Sozialgeschichte, Göttingen, S. 166-210.

Mackensen, R., 1979: Bevölkerung und Gesellschaft in Deutschland - Die Entwicklung 1945-1978, in: Matthes, J. (Hrsg.), Sozialer Wandel in Westeuropa. Verhandlungen des 19. Deutschen Soziologentages, Frankfurt: Campus, S. 433-464.

Merkatz, H. J. (Hrsg.), 1979: Aus Trümmern wurden Fundamente. Vertriebene, Flüchtlinge, Aussiedler. Drei Jahrzehnte Integration, Düsseldorf.

Mooser, J., 1983: Abschied von der „Proletarität“. Sozialstruktur und Lage der Arbeiterschaft in der Bundesrepublik in historischer Perspektive, in: Conze, W., Lepsius, M. R. (Hrsg.), Sozialgeschichte der Bundesrepublik Deutschland. Beiträge zum Kontinuitätsproblem, Stuttgart, S. 143-186.

Müller, W., Mayer, K. U.: Chancengleichheit durch Bildung? Untersuchungen über den Zusammenhang von Ausbildungsabschlüssen und Berufsstatus. Stuttgart 1976. Deutscher Bildungsrat: Gutachten und Studien der Bildungskommission, Bd. 42.

Narr, W. D., Tränhardt, D. (Hrsg.), 1979: Die Bundesrepublik Deutschland. Entstehung - Entwicklung Struktur, Königstein/Taunus.

Nellner, W., 1959: Grundlagen und Hauptergebnisse der Statistik, in: Lemberg, E., Edding, F. (Hrsg.), 1959, Band 1, S. 63-136. 
Rauch, P., 1979: Vertriebene und Nichtvertriebene im Vergleich, in: Merkatz (Hrsg.), S. 263-278.

Schillinger, R., 1985: Der Lastenausgleich, in: Benz, W. (Hg.): Die Vertreibung der Deutschen aus dem Osten, Frankfurt: Fischer, S. 183-192.

Schönberg, H. W., 1970: The Germans from the East. A study of their Migration, Resettlement and Subsequent Group History since 1945, The Hague.

Statistisches Bundesamt 1975: Fachserie A, Bevölkerung und Kultur, Reihe 4, Vertriebene und Flüchtlinge 1974, Stuttgart/Mainz: Kohlhammer.

Steiger, H./Tegtmeyer, H., 1975: Sozialstruktur im Wandel, in: Zeitschrift für Bevölkerungswissenschaft, 2, S. 14-38.
Tegtmeyer, H., 1976: Berufliche und soziale Umschichtung der Bevölkerung. Methodische Anmerkungen zur Planung, Durchführung und Aufbereitung der Befragung, in: Zeitschrift für Bevölkerungswissenschaft, 1, 1976, S. 34-55.

Vogelsang, T., 1975: Das geteilte Deutschland, München: dtv.

Waldemann, P., 1979: Die Eingliederung der ostdeutschen Vertriebenen in die westdeutsche Gesellschaft, in: Becker et al., S. 163-193.

Wallich, H. C., 1979: Das „Deutsche Wunder“ - eine Übersicht, in: Narr/Tränhardt (Hrsg.), S. 60-74. 